\title{
Bioinspired Musculoskeletal Model-based Soft Wrist Exoskeleton for Stroke Rehabilitation
}

\author{
Ning $\mathrm{Li}^{1,2,3}$, Tie Yang ${ }^{1,2}$, Yang Yang ${ }^{1,2}$, Peng $\mathrm{Yu}^{12^{* *}}$, Xiujuan Xue ${ }^{4}$, Xingang Zhao ${ }^{1,2}$, Guoli Song ${ }^{1,2}$, \\ Imad H. Elhajj ${ }^{5}$, Wenxue Wang ${ }^{1,2}$, Ning $\mathrm{Xi}^{6}$, Lianqing Liu ${ }^{1,2^{*}}$ \\ 1. State Key Laboratory of Robotics, Shenyang Institute of Automation, Chinese Academy of Sciences, Shenyang 110016, China \\ 2. Institutes for Robotics and Intelligent Manufacturing, Chinese Academy of Sciences, Shenyang 110169, China \\ 3. University of Chinese Academy of Sciences, Beijing 100049, China \\ 4. Rehabilitation Center for the Disabled, Shenyang 110015, China \\ 5. Vision and Robotics Lab, Department of Electrical and Computer Engineering, American University of Beirut, \\ Beirut 1107 2020, Lebanon \\ 6. Emerging Technologies Institute, Department of Industrial \& Manufacturing Systems Engineering, \\ University of Hong Kong, Pokfulam, Hong Kong 999077, China
}

\begin{abstract}
Exoskeleton robots have demonstrated the potential to rehabilitate stroke dyskinesia. Unfortunately, poor human-machine physiological coupling causes unexpected damage to human of muscles and joints. Moreover, inferior humanoid kinematics control would restrict human natural kinematics. Failing to deal with these problems results in bottlenecks and hinders its application. In this paper, the simplified muscle model and muscle-liked kinematics model were proposed, based on which a soft wrist exoskeleton was established to realize natural human interaction. Firstly, we simplified the redundant muscular system related to the wrist joint from ten muscles to four, so as to realize the human-robot physiological coupling. Then, according to the above human-like musculoskeletal model, the humanoid distributed kinematics control was established to achieve the two DOFs coupling kinematics of the wrist. The results show that the wearer of an exoskeleton could reduce muscle activation and joint force by $43.3 \%$ and $35.6 \%$, respectively. Additionally, the humanoid motion trajectories similarity of the robot reached $91.5 \%$. Stroke patients could recover $90.3 \%$ of natural motion ability to satisfy for most daily activities. This work provides a fundamental understanding on human-machine physiological coupling and humanoid kinematics control of the exoskeleton robots for reducing the post-stroke complications.
\end{abstract}

Keywords: stroke rehabilitation, simplified muscle model, soft wrist exoskeleton robot, bionic kinematics model

Copyright $(\odot)$ The author(s) 2020 .

\section{Introduction}

Robotic-aided neuro-rehabilitation is a clinically viable option to improve rehabilitation outcomes for stroke dyskinesia ${ }^{[1,2]}$ which can assist $90 \%$ of patients to regain motion ability ${ }^{[3]}$. Upper limb functions are important for Activities of Daily Living (ADLs), like self-feeding and drinking ${ }^{[4,5]}$, and joints closer to the limb end are the more difficult to recover according to rehabilitation medicine ${ }^{[6]}$. The motion ability of the wrist joint greatly affects the accuracy and stability of grasping. Therefore, exoskeleton robot for wrist rehabilitation could effectively improve rehabilitative outcomes. However, current exoskeletons, including rigid exoskeletons $^{[7-10]}$ and soft exoskeletons ${ }^{[11,12]}$ still face the common problem of poor human-robot physiological coupling and humanoid kinematics control.

Rigid exoskeletons are widely used in structural applications and carrying a heavy load of large joints ${ }^{[13,14]}$. Nevertheless, the problems of rigid exoskeletons, such as poor dynamic responses and misalignment of the motion axes, limit its application in the wrist joint. The Universal Haptic Drive (UHD) ${ }^{[5]}$ is for the rehabilitation of the wrist joint. However, the UHD only can assist joints to move the horizontal plane, which cannot meet the normal range of ADLs. This limitation was overcome by a wrist exoskeleton, RiceWrist ${ }^{[15,16]}$. This exoskeleton encompasses most of the natural hu-

\footnotetext{
*Corresponding author: Peng Yu, Lianqing Liu

E-mail: yupeng@sia.cn, lqliu@sia.cn
} 
man space. However, the heavy mechanism and fully enclosed structure affected the motion. For this problem, WRES ${ }^{[17]}$, driven by tendon lines, and SCRIPT6, driven by a steel leaf spring, were designed to simplify the structure. However, these robots showed poor adaptability to different individuals. NU-Wrist ${ }^{[18]}$ is a passive self-aligning wrist rehabilitation robot. However, the lack of a handle and forearm support causes inconvenience. Other robotics devices for wrist neurorehabilitation, such as MIT-MANUS ${ }^{[19]}$, READAPT ${ }^{[20]}$, wrist robot ${ }^{[21]}$, and Wrist Gimbal ${ }^{[22]}$ with large inertia mechanisms still would increase metabolic costs.

Soft exoskeletons have been studied widely to solve the problems of rigid exoskeletons, like poor dynamic responses and motion axes misalignment. The soft exoskeleton is like clothing, which could augment motion ability without restricting natural kinematics. Researchers have developed a variety of soft exoskeletons for motion assistance. A soft exoskeleton, Pneumatic Assist Wear, is designed to reduce a physical burden based on pneumatic soft actuator ${ }^{[23]}$. However, heavy and slow response of pneumatic control limits its application. To overcome this shortcoming, Exo-Glove Poly $^{[24]}$, a soft glove is driven by tension lines for assisting patients to grasp objects. Nevertheless, manual key results in complex operations, and the thumb lacking the active drive results in an unnatural motion. Moreover, Soft Exosuit ${ }^{[25-28]}$ could provide natural walking assistance based on Inertial Measurement Unit (IMU) signals. However, these exoskeletons lack the effect analysis on the human physiological state. Therefore, a soft upper-limb exoskeleton was designed for elbow assistance, considering the physiological and kinematics effects on arm movements ${ }^{[29-31]}$. However, the layout of tension lines does not combine the muscle distribution of the human motion system, which reduces human-robot compliance. Moreover, current researches on soft exoskeletons mainly focus on the lower, upper limbs and hand. Comparatively, research of soft wrist exoskeletons is lacking and overlooked, which affects the accuracy of grasping and self-care ability. Furthermore, previous studies of soft exoskeletons lack in-depth physiological analyses of the body's own musculoskeletal system. Therefore, wearers suffer from the poor human-robot physiological coupling.
Hence, in this paper, the simplified muscle model and bionic kinematics model were proposed, based on which a bionic soft wrist exoskeleton was established to overcome the poor human-robot physiological coupling and humanoid kinematics control for natural human interaction. The research work conducted an in-depth study on the wrist joint. In our previous work ${ }^{[32,33]}$, we have verified the feasibility of the 7 DOFs of the soft upper limb exoskeleton. Next, the simplified muscle model and bionic kinematics model will be extended to the upper limbs and whole-body exoskeleton robots.

\section{Bio-inspired analysis and modeling}

\subsection{Simplified model of human muscle system}

The human motion is a highly nonlinear system which not only causes the difficulty of motion control but also poses major challenges for structural design. Therefore, it is necessary to simplify the muscle system.

We selected the wrist joint for our preliminary study to verify the simplified muscle model. Previous wrist exoskeletons had large and rigid structure ${ }^{[34,35]}$, which could only facilitate rehabilitation training without daily wearing.

\subsubsection{Wrist kinematics}

The wrist kinematics includes two DOFs: wrist flexion/extension and wrist adduction/abduction. The annular motion is the compound of two DOFs.

1. The main muscles for wrist flexion include the Flexor Carpi Radialis (FCR), Flexor Carpi Ulnaris (FCU) and Palmar Longus (PL);

2. The main muscles for wrist extension include the Extensor Carpi Radialis Longus (ECRL), Extensor Carpi Brevis Radialis (ECBR) and Extensor Carpi Ulnaris (ECU).

3. The main muscles for wrist adduction muscles include the FCU, the ECU.

4. The main muscles for wrist abduction include the FCR, ECRL, ECBR.

\subsubsection{Muscle kinematics model simplification}

According to the standards of the Wake Forest School of Medicine Institutional Review Board following the ethical standards of the Helsinki Declaration of 1975, revised in 2000, five muscles (ECRL, ECBR, 
ECU, FCR, and FCU) are mainly responsible for the two DOFs. Meanwhile, the FCR and PL have the consistent action direction and both ends points, which have the similar driving effect. FCR plays the main role during wrist flexion relative to PL, which was simplified and the muscles (FCR and PL) are equivalent to a robust muscle to simplify the model. Combined with the above anatomical analysis and reference standards, we further simplified the human skeletal muscle system according to anatomy and biomechanics. The ECRL and ECBR were simplified into a single muscle owing to the equivalence of the motion effect and position overlap. Moreover, the muscle model was simulated and validated in the human skeletal muscle simulation software, Opensim ${ }^{[36]}$. The FCU, ECRL, FCR, and ECU work together to ensure the stability of the wrist joint movement.

\subsection{Biomechanical simulation analysis}

First, we use an optical motion capture system,
Vicon, to record the motion trajectories of healthy subjects. Then, the motion trajectories were imported into Opensim.

\subsubsection{Healthy subject motion analysis}

The wrist flexion/extension motions were selected as the reference motion data. The joint angles, muscle force, and joint force were obtained as shown in Figs. 1a-1d. In terms of joint angles in Fig. 1b, the angles of wrist flexion/extension change obviously, and the angle of wrist adduction/abduction hardly changed. It is consistent with the actual motion condition. In terms of muscle forces in Fig. 1c, the amplitude of FCR and FCU is large, which contributes to the main power for the movement. The ECU and ECRL have low amplitude. In the human motion system, muscle force curves indicate the cooperation and antagonistic effects. In terms of joint forces in Fig. 1d, the force curves of $X, Y$, and $Z$ axes also have obvious motion directivity and shows the direction consistent with the motion.

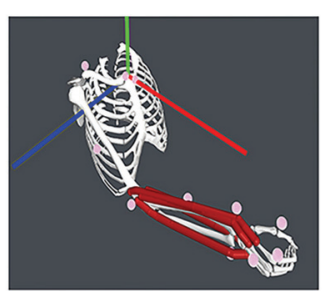

(a)

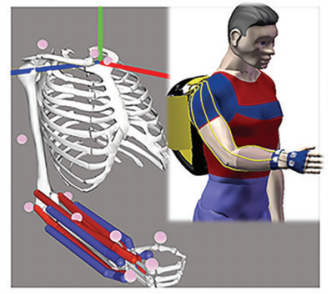

(e)

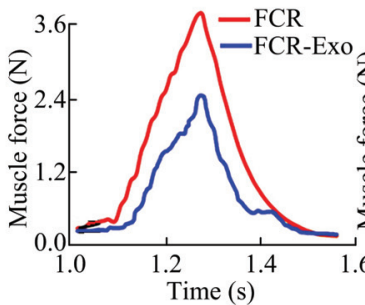

(i)

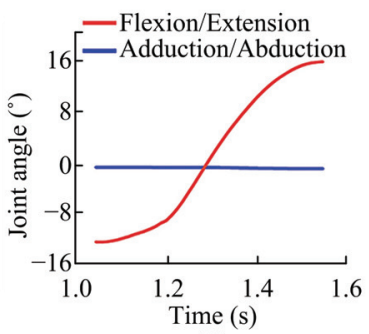

(b)

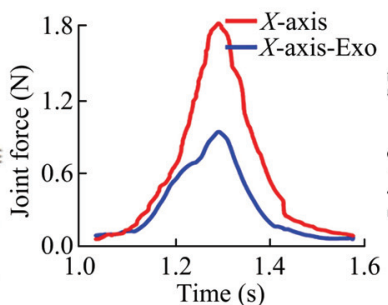

(f)

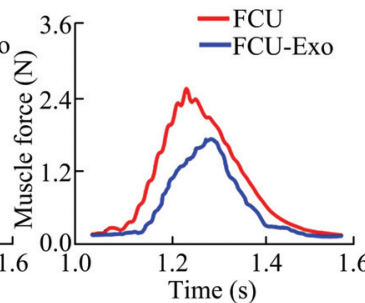

(j)

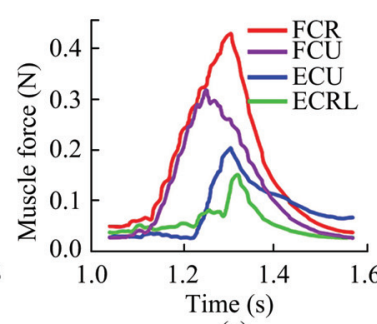

(c)

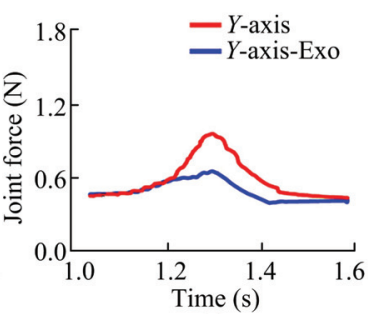

(g)

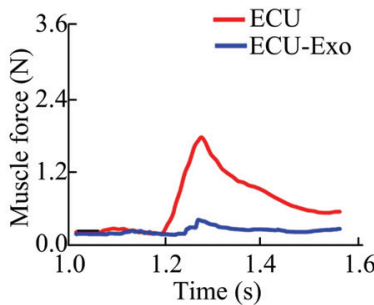

(k)

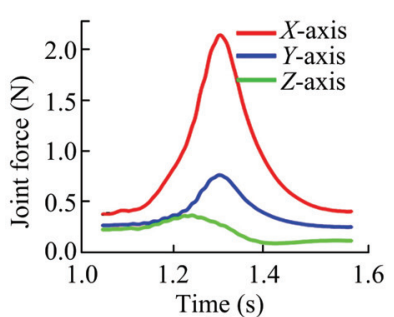

(d)

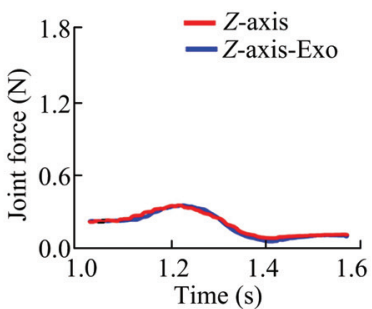

(h)

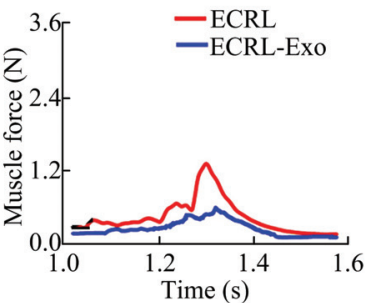

(l)

Fig. 1 The Opensim simulation results of human. (a-d) Motion data of healthy subject without exoskeleton assistance. (a) Distribution of four muscles (ECRL, FCU, ECU, FCR) and motion capture markers (Pink Points); (b) the angle curves; (c) the force curves; (d) the joint force curves. (e-l) Physiological results with exoskeleton assistance. (e) Human muscles (Red Lines) and exo-muscles (Blue Lines); (f-h) joint forces with and without assistance; (i-1) muscles force with and without assistance. 
Table 1 Physiological state of wrist joint before and after assistance

\begin{tabular}{ccc}
\hline Category & $\begin{array}{c}\text { The integral value of } \\
\text { muscles force }(\mathrm{N})\end{array}$ & $\begin{array}{c}\text { The integral value of } \\
\text { joint force }(\mathrm{N})\end{array}$ \\
\hline Before assistance & 78.0 & 359.5 \\
After assistance & 44.2 & 231.5 \\
Ratio of reduction & $43.3 \%$ & $35.6 \%$ \\
\hline
\end{tabular}

\subsubsection{Evaluation of exoskeleton physiological effects}

According to the simplified muscle model, four muscles (FCR, FCU, ECU, and ECRL) drive the wrist motion. The same state of wrist motion data was input into Opensim to evaluate the human physiological state. These simulation results are shown in Figs. 1e-11.

According to the simulation results, the muscle and joint forces significantly reduced by $43.3 \%$ and $35.6 \%$ respectively with exoskeleton assistance under the same motion state shown in Table 1. These results demonstrate that the simplified muscle model enables the human run in a low-power state without harm to the physiological structure.

\subsection{Bionic kinematics model}

The robot has the drive space, joint space and task space (Descartes Space). The mapping relations of three spaces are the basis of kinematics control. The articulated robot can achieve good kinematics control based on some traditional methods, such as DenavitHartenberg (DH) model. However, the robot in this study adopted the distributed muscle drive mode, whose drive space is not coincident with joint space. Therefore, the bionic kinematics model was proposed to solve the control problem for non-alignment of drive space and joint space.

First, we established the mapping between task space (hand) and joint space (joint angle) based on the DH model. Second, the mapping between the joint space (joint angle) and drive space (tension line) was developed based on the transformation of spatial relations. Finally, the above two mapping relations were combined to connect the above three spaces. Based on the above relationships, we can control the angle of the wrist joint by adjusting the tension line.

The soft exoskeleton robots itself don't have precise DH parameters due to the variability of soft material. But the skeletons of the wearers are rigid with the stable

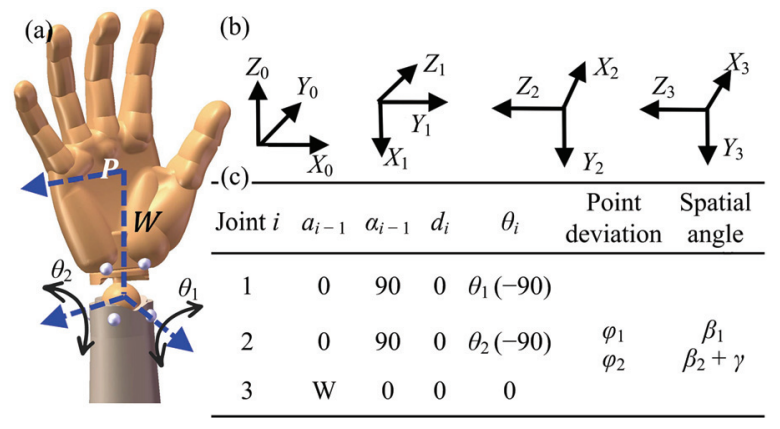

Fig. 2 The mapping of between hand and wrist joint. (a) The 3D model of the wrist with the DOFs; (b) the coordinate systems corresponding to the $3 \mathrm{DOFs}$; (c) DH parameters of the wrist and hand.

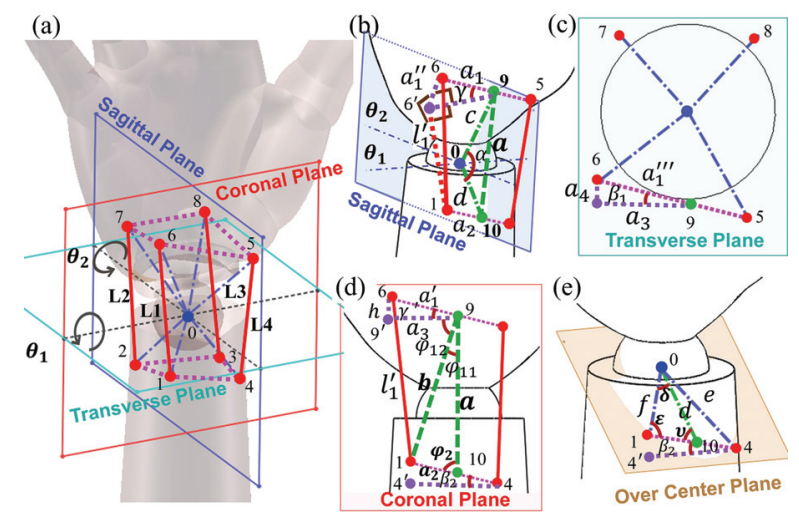

Fig. 3 Tension lines spatial configuration. (a) Bionic configuration model diagram; (b) axonometric view; (c) top view; (d) front view; (e) view of center rotation plane and the lower line $(1,4)$. Red points represent muscle nodes. Blue points represent the center point. The solid lines represent the actual lines, and the dotted lines represent the virtual auxiliary lines. Purple lines represent the auxiliary reference lines.

structure. Therefore, DH parameters of the robot could be determined based on the human skeleton, as shown in Figs. 2a-2c. Therefore, the relationship between the joint space and task space was obtained using the $\mathrm{DH}$ matrix as:

$$
\begin{gathered}
{ }_{0}^{3} \boldsymbol{T}={ }_{0}^{1} \boldsymbol{T}_{1}^{2} \boldsymbol{T}_{2}^{3} \boldsymbol{T}=\left[\begin{array}{cc}
{ }_{0}^{3} \boldsymbol{R} & { }_{0}^{3} \boldsymbol{P} \\
\mathbf{0} & \mathbf{1}
\end{array}\right], \\
{ }_{0}^{3} \boldsymbol{P}=\left[\begin{array}{l}
\boldsymbol{x}_{3} \\
\boldsymbol{y}_{3} \\
\boldsymbol{z}_{3}
\end{array}\right]=\left[\begin{array}{c}
\boldsymbol{W} \cos ^{2} \theta_{1} \\
-\boldsymbol{W} \sin \theta_{2} \\
\boldsymbol{W} \cos \theta_{1} \sin \theta_{1}
\end{array}\right],
\end{gathered}
$$

where $\boldsymbol{W}$ is center distance from palm to the wrist.

\subsubsection{Distribution of muscle tension lines}

According to above simplified muscle model, the starting and ending points of tension lines are arranged as shown in Fig. 3. 
Table 2 The symbolic index used in this study

\begin{tabular}{|c|c|c|c|c|c|}
\hline Symbol & Elucidation & Symbol & Elucidation & Symbol & Elucidation \\
\hline point 0 & the wrist center of rotation & $a_{1}^{\prime \prime}$ & $a_{1}$ from the side view & $\beta_{2}$ & angle of the line $(1,4)$ and horizon \\
\hline points $1-8$ & end positions of four muscles & $a_{1}^{\prime \prime \prime}$ & $a_{1}$ from the top view & $\gamma \& \gamma^{\prime}$ & $\begin{array}{l}\text { angle of the line }(6,7) \text { and horizon } \\
\& \gamma \text { in the coronal plane }\end{array}$ \\
\hline points $9-10$ & point of sagittal plane and line & $a_{3}$ & $a_{1}^{\prime \prime \prime}$ from the front view & $\varphi_{2}$ & angle of the sagittal line and $a_{3}$ \\
\hline$l_{1}$ & the length of the first pull line & $a_{4}$ & $a_{1}^{\prime \prime \prime}$ from the side view & $\varphi_{11}$ & angle of $b$ and coronal line \\
\hline$l_{1}^{\prime}$ & $l_{1}$ from the front view & $c$ & the line from point 9 to the center & $\varphi_{12}$ & the angle of $b$ and plane \\
\hline$a$ & line 9 and point 10 & $d$ & the line of point 10 to the center & $\delta$ & $\angle(1,0,10)$ in the triangle $(0,1,10)$ \\
\hline$b$ & the line of points 1 and 9 & $f$ & the line of points 1 and 0 & $\varepsilon$ & $\angle(1,0,10)$ of $f$ and $a_{2}$ \\
\hline$a_{1}$ & the line of points 7 and 9 & $e$ & the line between points 4 and 0 & $v$ & $\angle(1,0,10)$ of $d$ and $a_{2}$ \\
\hline$a_{2}$ & the line of points 1 and 10 & $\alpha_{1}$ & the angle between $c$ and $d$ & & \\
\hline$a_{1}^{\prime}$ & $a_{1}$ from the coronal plane & $\beta_{1}$ & angle of the line $(6,9)$ and horizon & & \\
\hline
\end{tabular}

The symbols used in this paper are listed in Table 2 with their definitions. The length of the $l_{1}$ could be obtained from Fig. 3 according to Eq. (3):

$$
\begin{aligned}
l_{1}^{2} & =a_{1}^{\prime \prime 2}+l_{1}^{\prime 2} \\
& =\left(a_{1} \sin \gamma\right)^{2}+\left(a_{1}^{\prime 2}+b^{2}-2 a_{1}^{\prime} b \cos \left(\varphi_{12}+\gamma^{\prime}\right)\right) .
\end{aligned}
$$

The variables $a_{1}, \gamma, b, \varphi_{12}, \gamma^{\prime}$, and $a_{1}^{\prime}$ in Eq. (3) need to be obtained. Among them, $a_{1}$ and $\gamma$ were initially calibrated attached to the system. The other variables $b, \varphi_{12}, \gamma^{\prime}$, and $a_{1}^{\prime}$ should be calculated according to the geometric relationships as:

$a$ is obtained by cosine theorem in the triangle $(0,9$, 10) of Fig. 3b. $b$ can be calculated based on the sine theorem by substituting $a$ into the triangle $(1,9,10)$ according to Fig. 3d in Eq. (4).

$$
\begin{aligned}
b^{2}= & \left(c^{2}+d^{2}-2 c d \cos \alpha\right)+a_{2}^{2} \\
& -2 a_{2} \cos \varphi_{2} \sqrt{c^{2}+d^{2}-2 c d \cos \alpha} .
\end{aligned}
$$

$\varphi_{12}$ is obtained by the angle relationship between parallel lines (line(4-4'), line(9-9')) between $\varphi_{11}, \varphi_{12}, \varphi_{2}$ and $\beta_{2}$ according to Fig. $3 d$ in Eq. (5).

$$
\varphi_{12}=\left(\pi-\varphi_{2}-\beta_{2}\right)-\arcsin \left(\frac{a_{2}}{b} \sin \varphi_{2}\right) .
$$

$\gamma^{\prime}$ and $a_{1}^{\prime}$ could be obtained from the triangle $(6,9$, $\left.9^{\prime}\right)$ in Fig. 3d.

$h$ is the constant value and from point 6 to the horizontal plane.

Moreover, the distances from the muscle nodes (1-8) to center (0) remain unchanged. The values of $a_{2}$ and $d$ would change during motion and could be obtained from the triangle $(0,1,10)$ in Fig. 3e.

$$
\zeta_{i}=\frac{\sin \psi_{i}}{\sin v} f
$$

where $\zeta_{1}=a_{2}, \psi_{1}=\delta, \zeta_{2}=d, \psi_{2}=\varepsilon$.

The above space conversion relation is the kinematics model imitating the human muscle configuration. In the model, the length of lines could be obtained by calibration and calculation. And, angles were calculated as follows:

$\beta_{1}, \beta_{2}$ and $\varphi_{2}$ are constants, which determined by the configuration of the robot and need to be identified at the eginning. Other angles varied with the DOFs of the joint angles $\theta_{1}$ and $\theta_{2}$.

$$
\left\{\begin{array}{l}
\varsigma_{i}=\varsigma_{i}^{0}+\Delta \theta_{1} \\
\alpha=\alpha^{0}+\Delta \theta_{2}
\end{array},\right.
$$

where $\varsigma_{1}=\varphi_{2}, \varsigma_{2}=\gamma, \varsigma_{3}=\delta, \varsigma_{4}=\varepsilon$. Among these parameters, $\varphi_{2}^{0}, \gamma^{0}, \delta^{0}$, and $\varepsilon^{0}$ are the initial value of the system, which can be calibrated initially. $v$ could be obtained from the triangle $(0,1,10)$ in Fig. 3e based on the $\delta^{0}$ and $\varepsilon^{0}$.

Based on the above parameter, the length of the controlled line could be obtained by Eq. (3) based on the $\theta_{1}$ and $\theta_{2}$. Therefore, the posture of the hand and wrist could be controlled based on the above model.

Circular motion of the bionic kinematics is shown in Figs. $4 a$ and $4 b$. The single DOF motion simulation is shown in Figs. $4 \mathrm{c}$ and $4 \mathrm{~d}$, and the motion trend of the four points can be seen in Fig. 4e and Fig. 4f, which is consistent with the actual motion pattern. The quadrilateral frame represents the wrist posture. Based on the above motion simulation, the validity of the bionic kinematics model was preliminarily verified. 


\section{System design}

\subsection{Physical humanoid platform design}

To better simulate the motion model, a bionic physical platform was designed and established with two
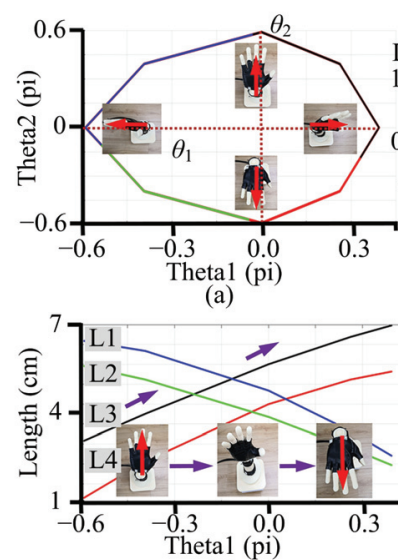

(c)

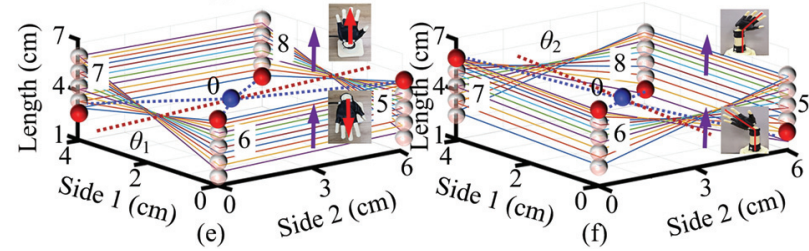

Fig. 4 Bionic kinematics simulation. (a, b) Circular motion: The length of lines varies during circular motion (four colors represent four tension lines). (c-f) Single-DOF: (c) The lines vary during wrist flexion/extension; (d) the lines vary during wrist adduction/abduction; (e) the wrist posture changing; (f) the wrist posture changing trend.
DOFs. The wrist joint was simplified as a spherical hinge joint in Fig. 4a. The motion platform was manufactured via 3D printing.

\subsection{System design}

According to the simplified muscle model, the locations of the tension endpoints were arranged. The force stressed structure was manufactured via 3D printing. And the soft structure composition of the exoskeleton robot used a commercial body protector (McDavid, Woodridge, Illinois, USA) as shown in Fig. 5 b.

The driver system was designed based on the EtherCAT bus protocol. A PC/104 computer and Maxon motors (Maxon Motor Ag., Sachseln, Switzerland) with Elmo drivers were used to implement position control. A $24 \mathrm{~V}$ power supply (SZ DJI Technology Co., Ltd., Shenzhen, China) was used to provide power to the system. Two wireless IMUs (Alubi, Guangzhou, China) recorded joint motion angles as shown in Figs. 5d and 5e. Furthermore, the ligament of the wrist joint was imitated to ensure that the tension lines fitted the human body.

\subsection{Control scheme}

First, we analyze the wrist posture to obtain the trajectories according to tasks. Then, the reference interpolation points were computed on the trajectory path. Next, the reference points were computed using inverse
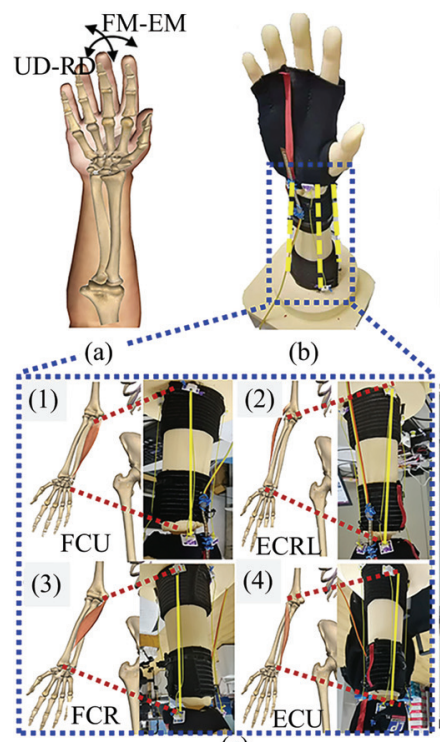

(c)

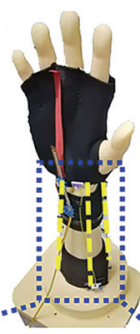

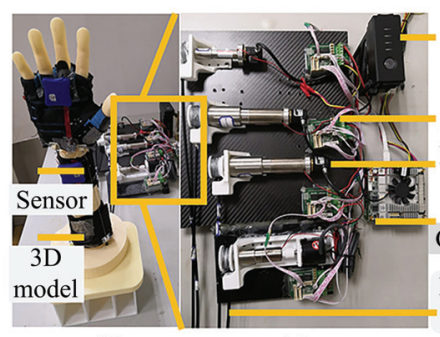

(e)

(d)

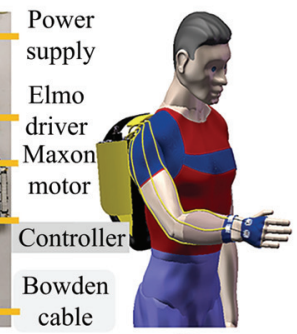

(f)

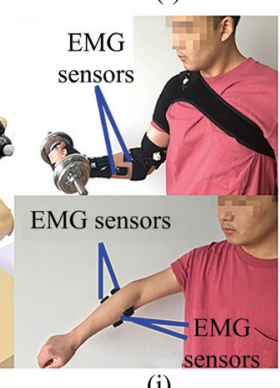

(h)

Fig. 5 Structural design and experimental equipment. (a) Anatomical structure of the wrist; (b) physical humanoid platform; (c1-c4) Maps of muscles and corresponding tension lines; (d) overall schematic diagram of the hardware; (e) the drive system; (f) conceptual diagram of power suit; (g, h) VICON: the optical motion capture system: (g) Healthy subject; (h) Stroke patient (Volunteer 1); (i) EMG (Electromyography) capture experiments. 


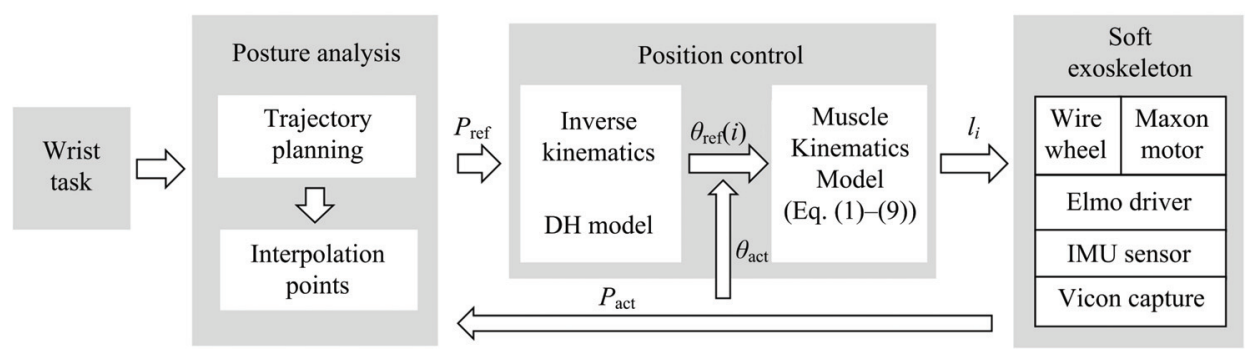

Fig. 6 Overall block diagram of wrist joint space control strategy.

kinematics to obtain the reference joint angles. These angles were calculated using bionic kinematics based on Eqs. (3)-(8) to obtain the tension line lengths. Further, the motors' rotation angles were calculated based on the line lengths and the wire wheel. Elmo drove the motors to realize robot motion control. Finally, wrist angles were recorded by the IMUs and fed back into the motion control system as shown in Fig. 6.

\subsection{Participants}

Three stroke (hemorrhagic) survivors ( 3 males, age range 32-57 years) were enrolled for an uncontrolled clinical trial. Table 3 provides the basic information for all the participants. This study was carried out in cooperation with the Rehabilitation Center for the Disabled of Liaoning Province (the fifth author's institution). All of the operations are following rehabilitation regulations and ethical norms. The post-stroke participants in the experiments gave informed consent for the study and for using their identifiable images.

\section{Results and discussion}

\subsection{Humanoid platform motion experiments}

In the motion trajectory experiments, the 3D motion capture system (VICON, Oxford Metrics, UK) was used to capture the marker points attached to the human body. Firstly, to verify the simplified muscle model and kinematics models, wrist flexion/extension, wrist adduction/abduction and circular motions were carried on the physical humanoid platform as shown in Figs. $5 \mathrm{~g}$ and $5 \mathrm{~h}$. The process involved in a circular motion is shown in Figs. $7 \mathrm{a}$ and $7 \mathrm{~b}$. The motion range of wrist joint is able to cover the daily living motion range of human wrist (wrist flexion/extension: $115^{\circ}$; and wrist adduction/abduction: $\left.70^{\circ}\right)^{[37]}$. It enables the proposed soft exoskeleton to achieve the similar motion as the previous rigid exoskeleton ${ }^{[38,39]}$. Meanwhile, the trajectory
Table 3 Basic information of volunteer stroke patients

\begin{tabular}{cccc}
\hline Serial number & age & Injured side & Injured side \\
\hline Volunteer 1 & 32 & Right & Shoulder/Elbow/Wrist \\
Volunteer 2 & 45 & Right & Shoulder/Elbow/Wrist \\
Volunteer 3 & 57 & Right & Elbow/Wrist \\
\hline
\end{tabular}

Table 4 EMG signal amplitude comparison with and without exoskeleton assistance

\begin{tabular}{ccc}
\hline $\begin{array}{c}\text { The integral value } \\
\text { of muscles force }\end{array}$ & Simulation result & Experimental result \\
\hline Before assistance & 78.0 & 89.4 \\
After assistance & 44.2 & 46.5 \\
Ratio of reduction & $43.3 \%$ & $48.5 \%$ \\
\hline
\end{tabular}

similarity experiment was carried out, and the results show that the motion average similarity to the healthy subjects is over $91.5 \%\left(x_{1}, y_{1}, z_{1}\right)$, as shown in Fig. 7 d.

\subsection{Healthy subject experiments}

Two types of experiments on healthy subjects were carried out: motion trajectory experiments and EMG signal experiments.

\subsubsection{Motion trajectory experiments}

The wrist motion trajectories of the healthy subjects were captured by VICON and reproduced as shown in Fig. 7b. The Pearson correlation coefficients were determined to evaluate the trajectory similarity between the human and the robot. The average correlation was $0.903\left(x_{2}, y_{2}, z_{2}\right)$ in Fig. 7 d. These results demonstrate that the robot has a high similarity motion ability with healthy subjects. Meanwhile, the soft exoskeleton has better comfort and portability for daily wearing. And the lightweight isotropic device is an important trend for daily activities ${ }^{[16]}$. Therefore, some soft wrist exoskeletons were developed. However, our proposed soft exoskeleton makes progress in the functional diversity ${ }^{[40]}$ and kinesthetic feedback ${ }^{[41]}$. 


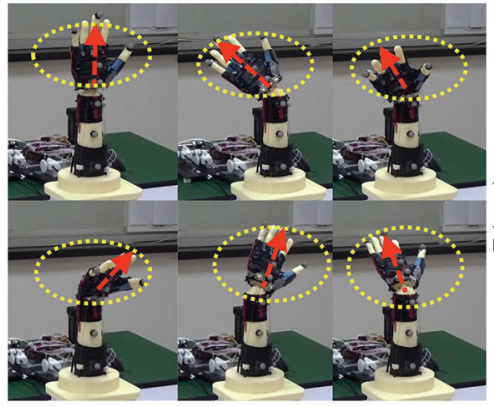

(a)

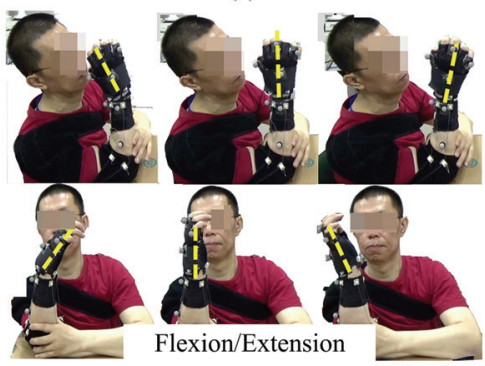

(e)

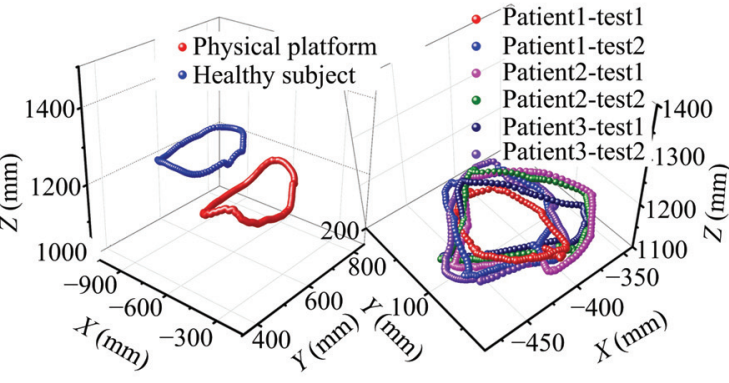

(b)

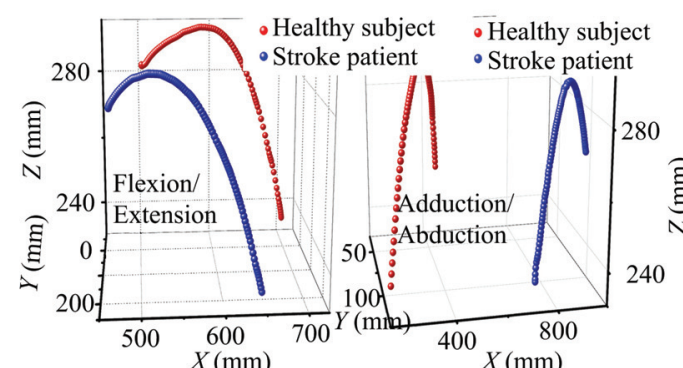

(g)

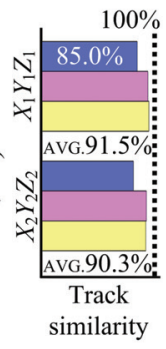

(d)

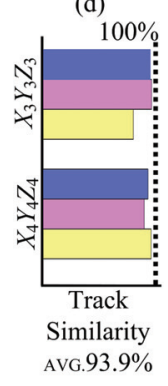

(h)

Fig. 7 Motion trajectories analysis. (a) Circular motion sequence; (b) circular motion comparison for the platform and the healthy subject; (c) repeated trajectory of three patient volunteers; (d) track similarity analysis: $(X, Y, Z)^{1}$ is for the platform and healthy subjects; $(X, Y, Z)^{2}$ is for stroke patients with the exoskeleton and healthy subjects; (e) single-DOF experiments. (Volunteer 2); (f, g) single-DOF trajectories: (f) wrist flexion/extension; (g) wrist adduction/abduction; (h) track similarity of healthy subjects and stroke patients: $(X, Y, Z)^{3}$ is for wrist adduction/abduction; $(X, Y, Z)^{4}$ is for wrist flexion/extension.

\subsubsection{EMG signal experiments}

The amplitude of the EMG (Electromyography) signals is positively related to the muscle output force at the Maximum Voluntary Isometric Contraction (MVIC) level. We used a Trigno ${ }^{\mathrm{TM}}$ Wireless System (Delsys, Inc., Natick, Massachusetts, USA) to capture the EMG signals. The decline in the EMG signal amplitude represents the assistance effect.

The EMG signal of healthy subjects was captured during lifting $5 \mathrm{~kg}$ weight during wrist flexion with and without exoskeleton assistance in Fig. 5i. The amplitudes of the EMG signals from four muscles (ECRL, FCU, ECU, and FCR) are shown in Fig. 8. These four muscles correspond to the muscles in our simplified muscle model. EMG could be the gold standard for the analysis of human muscle activation ${ }^{[42]}$, which the evaluated force assistance performance of exoskeleton. Some researches focus on the EMG variation before and after assistance as the input of control information ${ }^{[43,44]}$. The results show the EMG signal with assistance was reduced by $48.5 \%$ compared to that without assistance. The value is slightly lower than that of elbow joint ${ }^{[31,33]}$. It is because the main function of wrist joint relative to elbow joint is position adjustment function, not to carry

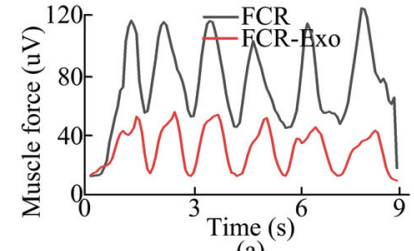

(a)

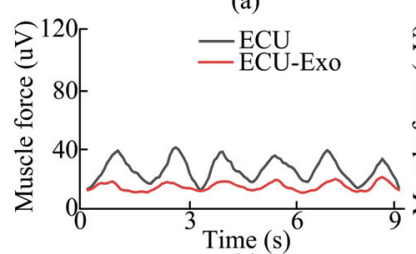

(c)

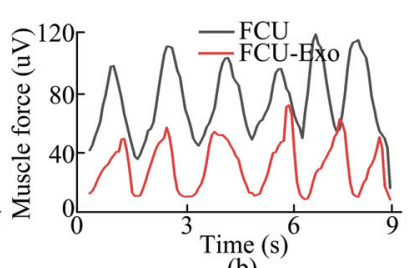

(b)

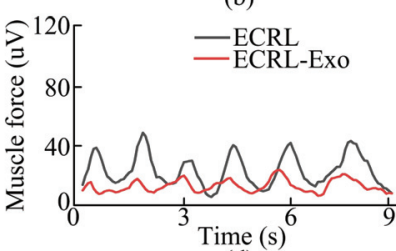

(d)
Fig. 8 The EMG activation degree of the healthy subjects with and without assistance. Grey Line: Without assistance; Red Line: With assistance.

the load. Therefore, in the force assistance experiment, the decreased EMG amplitude is slightly lower. In particular, this value is close to the above simulation value of $43.3 \%$ by Opensim in Table 4, which forms a closedloop verification of theory and experiment.

\subsection{Motion experiments on stroke patients}

Tests on stroke patients were carried out to verify the clinical assistance effect. The motion ability of these patients improved with the soft exoskeleton, and ADLs 
could be restored.

\subsubsection{Single-DOF motion experiment}

The patients in the experiment totally lost their wrist motion ability owing to stroke complications. The single-DOF motion was restored with exoskeleton assistance, as shown in Fig. 7e. The trajectories were recorded using the VICON system and reproduced as in Figs. $7 \mathrm{f}$ and $7 \mathrm{~g}$ ). The similarity between the patients and healthy subjects reaches $93.9 \%$ shown in Fig. 7 h. The average two DOFs motion range of patients recovered to $90.3 \%$ of the healthy subject shown in Table 5. The motion range is slightly lower than the rigid rehabilitation wrist exoskeleton ${ }^{[17]}$ due to deformation of soft structure. Here, the motion range could meet the need of ADLs. Further, the compensation algorithm and structure design are being carried out in the next step work to solve the problem of deformation and displacement.

\subsubsection{Daily activity experiment}

Drinking action was selected to evaluate the assistance effect of the exoskeleton in Fig. 9. The patient couldn't drink by wrist falling off $-65^{\circ}$. With assistance from the exoskeleton, he could move the cup to the mouth and drink by $20^{\circ}$ radially wrist bending in Figs. 9e-9h. These results prove the motion function recovery effect of the stroke patient with the exoskeleton in ADLs. And, most of current devices are large in size and need to be fixed and trained in specific positions ${ }^{[45]}$. The proposed exoskeleton is portable and could be worn when the wearers move around. And, the current wrist exoskeletons are mainly used to rehabilitation training ${ }^{[46]}$. The exoskeleton in this paper could assist the patients with activities of daily living before restoring their motion ability.

It's well known that rehabilitation is a long-term progress $^{[45]}$. A lightweight and comfortable exoskeleton robot with high degree of human-robot fusion can be better accepted by patients. The previous exoskeletons used to adopt the rigid structure, and, due to the irregular physiological structure of human joint, additional complex mechanisms need to be designed to ensure the alignment of human and robot joint axes ${ }^{[47]}$. Thus, this research mainly focuses on three parts: bionic configuration, bionic drive, and bionic control. Compared with previous research on exoskeleton robots, the
Table 5 The motion trajectory recovery of patients compared with healthy subject

\begin{tabular}{lccc}
\hline & \multicolumn{2}{c}{ The contrast of trajectory length increase (mm) } \\
\cline { 2 - 4 } & $\begin{array}{c}\text { Wrist flex- } \\
\text { ion/extension }\end{array}$ & $\begin{array}{c}\text { Wrist adduc- } \\
\text { tion/abduction }\end{array}$ \\
\hline Stroke No Exo & $\rightarrow 0$ & $\rightarrow 0$ \\
Patient $\quad$ Exo & 197 & 247 \\
Healthy Subject & 217 & 275 \\
Increase Range & $90.7 \%$ & $89.8 \%$ \\
Average Increase & & $90.3 \%$ \\
\hline
\end{tabular}

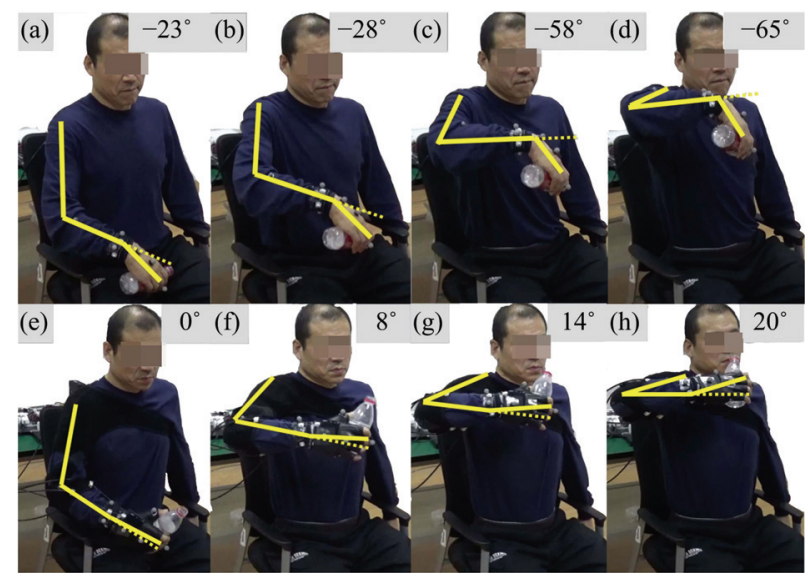

Fig. 9 Drinking water in comparison to the patient without and with the assistance (Volunteer 3). (a-e) The patient failed to drink water independently; ( $\mathrm{f}-\mathrm{j})$ the patient succeeded in drinking water with assistance. The angles in the figures represent the angle between the hand and the forearm.

proposed robot has the following innovative advantages:

(i) Bionic Configuration: Simplified muscle model: Based on anatomy and sport biomechanics, a simplified muscle system was discussed and proposed improving human-machine coupling and assistance efficiency based on the robust and flexible human muscle system.

(ii) Bionic Drive: Soft structure and compliant drive: The overall structure and drive part can be achieved by soft materials and tension lines, similar to human body flexibility based on the simplified muscle system according to biomechanics and anatomy.

(iii) Bionic Control: Bionic kinematics model: A bionic kinematics model was established to solve the problem that the drive space and joint space don't coincide in irregular distribution drive space, which realizes human-robot integration in the control strategy.

Besides, some limitations need to be overcome to further improve the performance of the robot in future studies, for example, the displacement and deformation caused by the soft structure. Compensation algorithm 
and more stable structure design will be carried out in the next step work. Furthermore, artificial intelligence should be combined to provide personalized assistance $^{[28,48-50]}$.

\section{Conclusion}

Human motion system is a nonlinear system with advantages. However, the complex and redundant system causes the difficulty of motion control and hardware design. In this study, the human muscle system was simplified to an executable model in a soft wrist exoskeleton. Meanwhile, a muscle-liked kinematics model was established to realize humanoid control, which solves the misalignment of drive space and joint space due to muscle-liked irregular distribution drive. The experimental results demonstrate that high-performance humanoid motion ability could improve the motion ability of stroke patients, which has the well-behaved human-robot coupling with the lower muscle activation and joint force. Thus, the proposed exoskeleton could be used in the process of stroke rehabilitation and daily life. And the methods of muscle simplification and bionics kinematic model could provide a thought for exoskeletons and humanoid robots.

\section{Acknowledgment}

This work was supported by National Key R\&D Program of China (Grant No. 2016YFE0206200), the National Natural Science of China (Grant Nos. 61821005, 61703395, and 61727811), the Sichuan Science and Technology Program (Grant No. 20SYSX0276), Natural Science Foundation of Liaoning Province of China (Grant No. 20180520035), and Youth Innovation Promotion Association of the Chinese Academy of Sciences (Grant No. 2019205).

Open Access This article is licensed under a Creative Commons Attribution 4.0 International License, which permits use, sharing, adaptation, distribution and reproduction in any medium or format, as long as you give appropriate credit to the original author(s) and the source, provide a link to the Creative Commons licence, and indicate if changes were made.

The images or other third party material in this article are included in the article's Creative Commons licence, unless indicated otherwise in a credit line to the material. If material is not included in the article's Creative Commons licence and your intended use is not permitted by statutory regulation or exceeds the permitted use, you will need to obtain permission directly from the copyright holder.

To view a copy of this licence, visit http://creativecommons.org/licenses/by/4.0/.

\section{References}

[1] Washabaugh E P, Claflin E S, Gillespie R B, Krishnan C. A novel application of eddy current braking for functional strength training during gait. Annals of Biomedical Engineering, 2016, 44, 2760-2773.

[2] Zheng T J, Zhu Y H, Zhang Z W, Zhao S K, Chen J, Zhao J. Parametric gait online generation of a lower-limb exoskeleton for individuals with paraplegia. Journal of Bionic Engineering, 2018, 15, 941-949.

[3] Elangovan N, Cappello L, Masia L, Aman J, Konczak J. A robot-aided visuo-motor training that improves proprioception and spatial accuracy of untrained movement. Scientific Reports, 2017, 7, 17054 .

[4] Hesse S, Schulte-Tigges G, Konrad M, Bardeleben A, Werner C. Robot-assisted arm trainer for the passive and active practice of bilateral forearm and wrist movements in hemiparetic subjects. Archives of Physical Medicine and Rehabilitation, 2003, 84, 915-920.

[5] Oblak J, Cikajlo I, Matjačić Z. Universal haptic drive: A robot for arm and wrist rehabilitation. IEEE Transactions on Neural Systems and Rehabilitation Engineering, 2010, 18, 293-302.

[6] Amirabdollahian F, Ates S, Basteris A, Cesario A, Buurke J, Hermens H, Hofs D, Johansson E, Mountain G, Nasr N, Nijenhuis S, Prange G, Rahman N, Sale P, Schätzlein F, Van Schooten B, Stienen A. Design, development and deployment of a hand/wrist exoskeleton for home-based rehabilitation after stroke-SCRIPT project. Robotica, 2014, 32, 1331-1346.

[7] He L, Xiong C H, Liu K, Huang J, He C, Chen W B. Mechatronic design of a synergetic upper limb exoskeletal robot and wrench-based assistive control. Journal of Bionic Engineering, 2018, 15, 247-259.

[8] Kim S, Lee J, Bae J. Analysis of finger muscular forces using a wearable hand exoskeleton system. Journal of Bionic Engineering, 2017, 14, 680-691.

[9] Shimizu Y, Kadone H, Kubota S, Suzuki K, Abe T, Ueno T, Soma Y, Sankai Y, Hada Y, Ya-mazaki M. Voluntary ambu- 
lation by upper limb-triggered hal ${ }^{\circledR}$ in patients with complete quadri/paraplegia due to chronic spinal cord injury. Frontiers in Neuroscience, 2017, 11, 649.

[10] Long Y, Du Z J, Chen C F, Wang W D, He L, Mao X W, Xu G Q, Zhao G Y, Li X Q, Dong W. Development and analysis of an electrically actuated lower extremity assistive exoskeleton. Journal of Bionic Engineering, 2017, 14, 272-283.

[11] Lessard S, Pansodtee P, Robbins A, Trombadore J M, Kurniawan S, Teodorescu M. A soft exosuit for flexible upper-extremity rehabilitation. IEEE Transactions on Neural Systems and Rehabilitation Engineering, 2018, 26, 1604-1617.

[12] Dinh B K, Xiloyannis M, Antuvan C W, Cappello L, Masia L. Hierarchical cascade controller for assistance modulation in a soft wearable arm exoskeleton. IEEE Robotics and Automation Letters, 2017, 2, 1786-1793.

[13] Rosales-Díaz I, López-Gutiérrez J R, Suárez A E Z, Salazar S R, Osorio-Cordero A, Aguilar-Sierra H, Lozano R. Comparison of control techniques in a weight lifting exoskeleton. Journal of Bionic Engineering, 2019, 16, 663-673.

[14] Shimizu Y, Kadone H, Kubota S, Ueno T, Sankai Y, Hada Y, Yamazaki M. Voluntary elbow extension-flexion using single joint hybrid assistive limb (HAL) for patients of spastic cerebral palsy: Two cases report. Frontiers in neurology, 2019, 10, 2.

[15] Abhishek G, Marcia K O M, Volkan P, Charles B. Design, control and performance of RiceWrist: A force feedback wrist exoskeleton for rehabilitation and training. International Journal of Robotics Research, 2008, 27, 233-251.

[16] Rose C G, Pezent E, Kann C K, Deshpande A D, O'malley M $\mathrm{K}$. Assessing wrist movement with robotic devices. IEEE Transactions on Neural Systems and Rehabilitation Engineering, 2018, 26, 1585-1595.

[17] Buongiorno D, Sotgiu E, Leonardis D, Marcheschi S, Solazzi M, Frisoli A. WRES: A novel 3 DoF wrist exoskeleton with tendon-driven differential transmission for neuro-rehabilitation and teleoperation. IEEE Robotics and Automation Letters, 2018, 3, 2152-2159.

[18] Omarkulov N, Telegenov K, Zeinullin M, Tursynbek I, Shintemirov A. Preliminary mechanical design of NU-Wrist: A 3-DOF self-aligning wrist rehabilitation robot. Proceedings of 2016 6th IEEE International Conference on Biomedical Robotics and Biomechatronics (BioRob), 2016, 962-967.

[19] Krebs H I, Volpe B T, Williams D, Celestino J, Charles S K, Lynch D, Hogan N. Robot-aided neurorehabilitation: A ro- bot for wrist rehabilitation. IEEE Transactions on Neural Systems and Rehabilitation Engineering, 2007, 15, 327-335.

[20] Rose C G, Sergi F, Yun Y, Madden K, Deshpande A D, Malley M K O. Characterization of a hand-wrist exoskeleton, readapt, via kinematic analysis of redundant pointing tasks. Proceedings of 2015 IEEE International Conference on Rehabilitation Robotics (ICORR), 2015, 205-210.

[21] Squeri V, Masia L, Giannoni P, Sandini G, Morasso P. Wrist rehabilitation in chronic stroke patients by means of adaptive, progressive robot-aided therapy. IEEE Transactions on Neural Systems and Rehabilitation Engineering, 2014, 22, 312-325.

[22] Martinez J A, Ng P, Lu S, Campagna M S, Celik O. Design of Wrist Gimbal: A forearm and wrist exoskeleton for stroke rehabilitation. Proceedings of 2013 IEEE 13th International Conference on Rehabilitation Robotics (ICORR), 2013, 6650459 .

[23] Kang B B, Lee H, In H, Jeong U, Chung J, Cho K. Development of a polymer-based ten-don-driven wearable robotic hand. Proceedings of 2016 IEEE International Conference on Robotics and Automation (ICRA), 2016, 3750-3755.

[24] Kang B B, Choi H, Lee H, Cho K-J. Exo-Glove Poly ii: A polymer-based soft wearable robot for the hand with a tendon-driven actuation system. Soft Robotics, 2018, 6, 214-227.

[25] Asbeck A T, De Rossi S M M, Holt K G, Walsh C J. A biologically inspired soft exosuit for walking assistance. The International Journal of Robotics Research, 2015, 34, 744-762.

[26] Awad L N, Bae J, O'donnell K, De Rossi S M M, Hendron K, Sloot L H, Kudzia P, Allen S, Holt K G, Ellis T D, Walsh C J. A soft robotic exosuit improves walking in patients after stroke. Science Translational Medicine, 2017, 9, eaai9084.

[27] Quinlivan B T, Lee S, Malcolm P, Rossi D M, Grimmer M, Siviy C, Karavas N, Wagner D, Asbeck A, Galiana I, Walsh C J. Assistance magnitude versus metabolic cost reductions for a tethered multiarticular soft exosuit. Science Robotics, 2017, 2, eaah4416.

[28] Ding Y, Kim M, Kuindersma S, Walsh C J. Humanin-the-loop optimization of hip assistance with a soft exosuit during walking. Science Robotics, 2018, 3, eaar5438.

[29] Dinh B K, Cappello L, Xiloyannis M, Masia L. Position control using adaptive backlash compensation for bowden cable transmission in soft wearable exoskeleton. Proceedings of 2016 IEEE/RSJ International Conference on Intelligent Robots and Systems (IROS), 2016, 5670-5676.

[30] Dinh B K, Xiloyannis M, Cappello L, Antuvan C W, Yen 
S-C, Masia L. Adaptive backlash compensation in upper limb soft wearable exoskeletons. Robotics and Autonomous Systems, 2017, 92, 173-186.

[31] Xiloyannis M, Chiaradia D, Frisoli A, Masia L. Physiological and kinematic effects of a soft exosuit on arm movements. Journal of NeuroEngineering and Rehabilitation, 2019, 16, 29.

[32] Li N, Yu P, Yang T, Zhao L, Liu Z W, Xi N, Liu L Q. Bio-inspired wearable soft upper-limb exo-skeleton robot for stroke survivors. Proceedings of 2017 IEEE International Conference on Robotics and Biomimetics (ROBIO), 2017, 2693-2698.

[33] Li N, Yang T, Yu P, Chang J L, Zhao L, Zhao X G, Elhajj I H, Xi N, Liu L Q. Bio-inspired upper limb soft exoskeleton to reduce stroke-induced complications. Bioinspiration \& Biomimetics, 2018, 13, 066001.

[34] Esmaeili M, Guy S, Dailey W D, Burdet E, Campolo D. Subject-specific wrist model calibration and application to ergonomic design of exoskeletons. IEEE Sensors Journal, 2013, 13, 3293-3301.

[35] Saul K R, Hu X, Goehler C M, Vidt M E, Daly M, Velisar A, Murray W M. Benchmarking of dynamic simulation predictions in two software platforms using an upper limb musculoskeletal model. Computer Methods in Biomechanics \& Biomedical Engineering, 2015, 18, 1445-1458.

[36] Delp S L, Anderson F C, Arnold A S, Loan P, Habib A, John C T, Guendelman E, Thelen D G. Opensim: Open-source software to create and analyze dynamic simulations of movement. IEEE Transactions on Biomedical Engineering, 2007, 54, 1940-1950.

[37] Erwin A, O'malley M K, Ress D, Sergi F. Kinesthetic feedback during 2DOF wrist movements via a novel MR-compatible robot. IEEE Transactions on Neural Systems and Rehabilitation Engineering, 2017, 25, 1489-1499.

[38] Lee J, Song B W, Yang W. Design of exoskeleton-type wrist human-machine interface based on over-actuated coaxial spherical parallel mechanism. Advances in Mechanical Engineering, 2018, 10, 1687814017753896.

[39] Pehlivan A U, Lee S, Malley M K O. Mechanical design of RiceWrist-S: A forearm-wrist exoskeleton for stroke and spinal cord injury rehabilitation. Proceedings of 2012 4th IEEE RAS \& EMBS International Conference on Biomedical Robotics and Biomechatronics (BioRob), 2012, 1573-1578.

[40] Choi H, Kang B B, Jung B K, Cho K J. Exo-wrist: A soft tendon-driven wrist-wearable robot with active anchor for dart-throwing motion in hemiplegic patients. IEEE Robotics and Automation Letters, 2019, 4, 4499-4506.

[41] Skorina E H, Luo M, Onal C D. A soft robotic wearable wrist device for kinesthetic haptic feedback. Frontiers in Robotics and $A I, 2018, \mathbf{5}, 83$.

[42] Gazzoni M, Celadon N, Mastrapasqua D, Paleari M, Margaria V, Ariano P. Quantifying forearm muscle activity during wrist and finger movements by means of multi-channel electromyography, PLOS ONE, 2014, 9, e109943.

[43] Khokhar Z O, Xiao Z G, Menon C. Surface EMG pattern recognition for real-time control of a wrist exoskeleton. BioMedical Engineering OnLine, 2010, 9, 41.

[44] Gopura R A R C, Kiguchi K. A human forearm and wrist motion assist exoskeleton robot with emg-based fuzzy-neuro control. Proceedings of 2008 2nd IEEE RAS \& EMBS International Conference on Biomedical Robotics and Biomechatronics, 2008, 550-555.

[45] Mazzoleni S, Tran V, Dario P, Posteraro F. Wrist robot-assisted rehabilitation treatment in subacute and chronic stroke patients: From distal-to-proximal motor recovery. IEEE Transactions on Neural Systems and Rehabilitation Engineering, 2018, 26, 1889-1896.

[46] Mcdaid A J. Development of an anatomical wrist therapy exoskeleton (AW-TEx). Proceedings of 2015 IEEE International Conference on Rehabilitation Robotics (ICORR), Singapore, Singapore, 2015, 434-439.

[47] Vitiello N, Lenzi T, Roccella S, Rossi S M M D, Cattin E, Giovacchini F, Vecchi F, Carrozza M C. Neuroexos: A powered elbow exoskeleton for physical rehabilitation. IEEE Transactions on Robotics, 2013, 29, 220-235.

[48] Marini F, Squeri V, Cappello L, Morasso P, Riva A, Doglio L, Masia L. Adaptive wrist robot training in pediatric rehabilitation. Proceedings of 2015 IEEE International Conference on Rehabilitation Robotics (ICORR), 2015, 175-180.

[49] Lam C, Yang A Y, Driggs-Campbell K, Bajcsy R, Sastry S S. Improving human-in-the-loop decision making in multi-mode driver assistance systems using hidden mode stochastic hybrid systems. Proceedings of 2015 IEEE/RSJ International Conference on Intelligent Robots and Systems (IROS), Hamburg, Germany, 2015, 5776-5783.

[50] Zhang J, Fiers P, Witte K A, Jackson R W, Poggensee K L, Atkeson C G, Collins S H. Human-in-the-loop optimization of exoskeleton assistance during walking. Science, 2017, 356, 1280-1284. 